\title{
How to Remedy Incorrect Duplicates in the CSD?
}

\section{Frank Fronczek ffroncz@lsu.edu}

Several cases will be presented in which correct structures in the Cambridge database are duplicated with corresponding structures which are erroneous (misidentified atoms, misplaced $\mathrm{H}$ atoms, etc.) and have been assigned different refcodes. Trans-tetraaqua-bis(isonicotinato)-copper(II), XAXFUX has over ten correct entries in the CSD, but FINXUY is the same with a misplaced $\mathrm{H}$ atom. $1 \mathrm{H}-$ imidazol-3-ium 4-hydroxybenzoate, HAGWAP has two correct entries in the CSD, but XUBSUJ is the same with misidentified $\mathrm{C}$ and $\mathrm{N}$ atoms and a misplaced $\mathrm{H}$ atom. The intensity data are available for these, and the errors are easily proven.

Bis((muz-hydroxo)-(2,2'-bipyridyl)-nitrato-copper(II)), BPYHCU has several correct entries, but WEXKIV is almost certainly the same with nitrate mistakenly treated as acetate and hydroxo mistakenly as oxo. Its intensity data are not available, so how to prove it? And when such proof is possible, what it the best way to make the correction in the database? Is a CSD Communication containing the new refinement using the original authors' data sufficient? 